\title{
Cure of prediabetic mice by viral infections involves lymphocyte recruitment along an IP-10 gradient
}

\author{
Urs Christen, ${ }^{1,2}$ Dirk Benke, ${ }^{3}$ Tom Wolfe, ${ }^{1,2}$ Evelyn Rodrigo, ${ }^{1}$ Antje Rhode, ${ }^{1}$ \\ Anna C. Hughes, ${ }^{2}$ Michael B.A. Oldstone, ${ }^{2}$ and Matthias G. von Herrath ${ }^{1}$ \\ ${ }^{1}$ Department of Developmental Immunology, La Jolla Institute for Allergy and Immunology, San Diego, California, USA \\ ${ }^{2}$ Department of Neuropharmacology, The Scripps Research Institute, La Jolla, California, USA \\ ${ }^{3}$ Institute for Molecular Medicine and Experimental Immunology, University of Bonn, Bonn, Germany
}

\begin{abstract}
Viruses can cause but can also prevent autoimmune disease. This dualism has certainly hampered attempts to establish a causal relationship between viral infections and type 1 diabetes (T1D). To develop a better mechanistic understanding of how viruses can influence the development of autoimmune disease, we exposed prediabetic mice to various viral infections. We used the well-established NOD and transgenic RIP-LCMV models of autoimmune diabetes. In both cases, infection with the lymphocytic choriomeningitis virus (LCMV) completely abrogated the diabetic process. Interestingly, such therapeutic viral infections resulted in a rapid recruitment of $\mathrm{T}$ lymphocytes from the islet infiltrate to the pancreatic draining lymph node, where increased apoptosis was occurring. In both models this was associated with a selective and extensive expression of the chemokine IP-10 (CXCL10), which predominantly attracts activated T lymphocytes, in the pancreatic draining lymph node, and in RIP-LCMV mice it depended on the viral antigenic load. In RIP-LCMV mice, blockade of TNF- $\alpha$ or IFN- $\gamma$ in vivo abolished the prevention of T1D. Thus, virally induced proinflammatory cytokines and chemokines can influence the ongoing autoaggressive process beneficially at the preclinical stage, if produced at the correct location, time, and levels.
\end{abstract}

J. Clin. Invest. 113:74-84 (2004). doi:10.1172/JCI200417005.

\section{Introduction}

Since development of type 1 diabetes (T1D) exhibits significant discordance in monozygotic twins carrying diabetes susceptibility MHC alleles (1-3), environmental factors such as infectious agents have been implicated as possible causes (4-8). However, attempts to establish a direct epidemiological association between viral infections and various autoimmune disorders have been unsuccessful (9). One of the reasons for this failure is the fact that viruses can enhance as well as abrogate autoimmune processes (10-17). While several viral infections have been associated with diabetes in northern countries (4-8), the incidence of both T1D and MS is reduced in equatorial countries, where microbial infections are more frequent $(1-3,9)$. Since each individual has frequently incurred multiple different viral infections by the time the autoimmune dis-

Received for publication September 27, 2002, and accepted in revised form October 27, 2003.

Address correspondence to: Matthias G. von Herrath, Department of Immune Regulation, IR-3, La Jolla Institute for Allergy and Immunology, 10355 Science Center Drive, San Diego, California 92121, USA. Phone: (858) 558-3571;

Fax: (858) 558-3579; E-mail: matthias@liai.org.

Conflict of interest: The authors have declared that no conflict of interest exists.

Nonstandard abbreviations used: type 1 diabetes (T1D); lymphocytic choriomeningitis virus (LCMV); pancreatic draining lymph node (PDLN); nucleoprotein (NP); rat insulin promoter (RIP); Armstrong (Arm); Pasteur (Past); glycoprotein (GP); intraperitoneal(ly) (i.p.); precursor CTL (pCTL); IFN- $\gamma$-inducible protein 10 (IP-10). ease is diagnosed (9), this dual effect likely hampers establishment of a causal association. In addition, positive and negative effects could create an equilibrium that is only rarely disturbed, resulting in disease in relatively few individuals. As a consequence, the pattern of viral infections encountered could be similar in nondiabetic compared with diabetic genetically predisposed patients. It is well described that viral infections can enhance autoimmunity via bystander activation (18) or molecular mimicry $(4-8,19,20)$. However, not much is known about how they prevent autoimmune diabetes. Studies in humans have been hampered by the fact that immune-mediated damage or modulation might occur after the causative viral infection has been cleared (a "hit and run" event) so that no viral "footprints" would be easily detectable in the affected organ(s). The purpose of the present study was twofold. First, we wished to examine whether viral infections could negatively influence an ongoing autoimmune process. Second, we wished to better understand mechanistically how islet destruction is abrogated.

We chose the well-established NOD mouse model of spontaneous diabetes (21) and the RIP-LCMV transgenic mouse model of virally induced autoimmune diabetes $(22,23)$. RIP-LCMV mice express the lymphocytic choriomeningitis virus (LCMV) nucleoprotein (NP) as a target autoantigen in their $\beta$ cells under control of the rat insulin promoter (RIP). The line used here offered the unique feature that clinical diabetes ( $>90 \%$ incidence) only develops 2 months after triggering of the autoimmune process with LCMV infection (24). Simi- 
lar to human T1D, disease in this murine model depends on both CD4 and CD8 lymphocytes, and autoantibodies to islet antigens are detectable prior to the onset of clinically overt diabetes (25). The 2-month window between viral (LCMV) trigger and disease development made this model ideally suited for testing the influence of sequential viral infections in a preclinical (prediabetic) state, when pancreatic insulin levels still suffice to maintain normoglycemia but the autoimmune process has already been initiated. Further, the autoaggressive response is easily traceable and quantifiable in this model, because the immunologically relevant LCMV-NP CD4 and CD8 T cell epitopes that cause disease have been defined (26-28).

In both models, we found that virally induced expression of IFN- $\gamma$-inducible protein 10 (IP-10, CXCL10) is higher in the pancreatic draining lymph node (PDLN) than in the islets. The expression of IP-10 in the PDLN is associated with a rapid egress of infiltrating lymphocytes that are usually present during the prediabetic insulitis. As a consequence, disease progression is halted and islet infiltration is reduced, leading to prevention of disease.

\section{Methods}

Breeding scheme and origin of mice. Generation of $\mathrm{H}-2^{\mathrm{b}}$ RIP-LCMV-NP transgenic mice has been described previously $(23,24)$. Mice were genotyped by PCR and Southern blotting as described by us previously (24). NOD/LtJ mice were purchased from The Jackson Laboratory (Bar Harbor, Maine, USA).

Virus. We used LCMV strains Armstrong (Arm) and Pasteur (Past) (29), and an LCMV escape variant (NPvar) selected not to recognize the $\mathrm{H}-2 \mathrm{D}^{\mathrm{b}}$-restricted NP epitope (30). This variant had a mutation in the immunodominant NP CTL epitope AA396-404 with a Phe-to-Leu change in residue 403, which completely abrogated the CTL response to NP in vitro and in vivo but allowed for a normal response to the two immunodominant glycoprotein (GP) CTL epitopes AA33-41 and AA276-286. The viruses were plaque-purified three times on Vero cells, and stocks were prepared by a single passage on BHK-21 cells. To trigger diabetes, RIPLCMV-NP mice were infected with a single intraperitoneal (i.p.) dose of $10^{5}$ PFUs of LCMV-Arm unless indicated otherwise.

Blood glucose measurements. Blood samples were obtained from the retro-orbital plexus, and plasma glucose concentration was determined using OneTouch Ultra (LifeScan, Milpitas, California, USA). Mice with blood glucose values higher than $300 \mathrm{mg} / \mathrm{dl}$ were considered diabetic (31).

RNase protection assay. Total RNA was isolated from whole-pancreas homogenates using TRI Reagent (Molecular Research Center Inc., Cincinnati, Ohio, USA). RNA was extracted with chloroform, subjected to isopropanol precipitation, and washed with ethanol. Twenty micrograms of total pancreatic RNA or PDLN was used for hybridization with a ${ }^{32} \mathrm{P}$-UTP-labeled multitemplate set provided by a commercial kit (RiboQuant, mCK-5c; Pharmingen, La Jolla, California, USA). The RNase protection assay was carried out according to the manufacturer's guidelines. The resulting analytical acrylamide gel was scanned using a Storm 860 PhosphorImaging system (Molecular Dynamics, Sunnyvale, California, USA), and the intensity of bands corresponding to protected mRNAs was quantified with ImageQuant image-analysis software (Molecular Dynamics) using L32 as a reference gene.

Cytotoxicity precursor CTL assays. For analysis of precursor frequency, splenic lymphocytes were diluted serially and cultivated in 96-well flat-bottom plates in the presence of $\mathrm{T}$ cell growth factor, LCMV-infected macrophages (irradiated with $20 \mathrm{~Gy}$ ), and syngeneic irradiated spleen-feeder cells or syngeneic feeder cells coated with $10 \mu \mathrm{M}$ LCMV NP ${ }_{396} \mathrm{H}-2 \mathrm{D}^{\mathrm{b}}$ peptide (FQPQNGQFI) (10 $0^{5}$ per well), as described (31). After 5-9 days, each well was assayed for CTL lysis (described above) on target cells that were uninfected, LCMVinfected, or LCMV NP ${ }_{396} \mathrm{H}-2 \mathrm{D}^{\mathrm{b}}$-peptide coated. The fraction of negative cultures (those with percentage lysis less than three SEs above background lysis of uninfected target cells) was determined for each dilution and correlated on a semilogarithmic scale with the number of splenocytes per well. The number of precursor CTLs (pCTLs) was determined according to the formula: $\mathrm{PCTL}=[4.6-\ln (\%$ negative wells $)] /$ number of splenocytes per well.

Immunohistochemistry. Organs were harvested at the times indicated, immersed in Tissue-Tek OCT compound (Sakura Finetek USA Inc., Torrance, California, USA), and quick-frozen on dry ice. Six- to ten-micrometer tissue sections were cut using a cryomicrotome and sialin-coated Superfrost Plus slides (Fisher Scientific Co., Pittsburgh, Pennsylvania, USA). Sections were fixed with $90 \% \mathrm{EtOH}$ at $-20^{\circ} \mathrm{C}$ and washed in PBS, and then avidin-biotin was blocked using the avidin/biotin blocking kit from Vector Laboratories Inc. (Burlingame, California, USA). Primary and biotinylated secondary antibodies (Vector Laboratories Inc.) were incubated with the sections for 30 minutes each, and color reaction was obtained by sequential incubation with avidin-peroxidase conjugate (Vector Laboratories Inc.) and diaminobenzidine-hydrogen peroxide. The primary antibodies used were rat anti-mouse $\operatorname{CD} 8 \alpha$ (ly2), rat anti-mouse $\operatorname{CD} 8 \beta$ (ly3), rat anti-mouse ICAM-1 (all from Pharmingen, San Diego, California, USA), and rat anti-mouse MHC class I (Bachem Biochemicals Inc., King of Prussia, Pennsylvania, USA).

Staining for apoptosis (TUNEL). Quick-frozen 6- to 10$\mu \mathrm{m}$ sections of pancreas and PDLNs were probed for the presence of apoptotic cells with a basic TUNEL assay using the ApopTag peroxidase in situ apoptosis detection kit (Intergen Co., Purchase, New York, USA) according to the manufacturer's guidelines. Briefly, TUNEL assay detects DNA strand breaks that are characteristic of the DNA fragmentation that occurs during apoptosis. Tissue sections were fixed with $1 \%$ 
paraformaldehyde and ethanol/acetic acid (2:1), washed, and incubated with terminal deoxynucleotidyl transferase to label free $3^{\prime} \mathrm{OH}$ DNA termini with digoxigenin-dNTP. Incorporated digoxigenin-dNTP was detected by sequential incubation with peroxidase-conjugated anti-digoxigenin $\mathrm{mAb}$ and diaminobenzidine-hydrogen peroxide.

Staining for apoptosis (flow cytometry). LCMV-NP- and LCMV-GP-specific cells were identified using $\mathrm{H}-2 \mathrm{D}^{\mathrm{b}}\left(\mathrm{NP}_{396}\right)$-tetramers and $\mathrm{H}-2 \mathrm{D}^{\mathrm{b}}\left(\mathrm{GP}_{33}\right)$-tetramers, respectively. MHC class I tetramers were generated using the immunodominant $\mathrm{H}-2 \mathrm{D}^{\mathrm{b}}$-restricted LCMV peptides $\mathrm{NP}_{396-404}$ (FQPQNGQFI) and $\mathrm{GP}_{33-41}$ (KAVYNFATC) for refolding and biotin-protein ligase BirA (Avidity, Denver, Colorado, USA) for biotinylation of the refolded MHC-peptide complexes using previously described procedures $(32,33)$. Biotinylated MHC-peptide complexes were tetramerized with allophycocyanin-conjugated streptavidin (Molecular Probes Inc., Eugene, Oregon, USA). Cells were stained with a FITC-conjugated anti-CD8 $\mathrm{mAb}$ (Pharmingen, La Jolla, California, USA), with allophycocyanin-conjugated $\mathrm{H}-2 \mathrm{D}^{\mathrm{b}}$-tetramers, and, for apoptosis, with phycoerythrin-conjugated annexin V (Pharmingen, La Jolla, California, USA) according to the manufacturer's guidelines. Cells were acquired and analyzed on a FACSCalibur flow cytometer using CellQuest software (Becton Dickinson Biosciences, San Jose, California, USA). For assessment of apoptosis, LCMV-specific CD8 lymphocytes were gated for CD8 ${ }^{\text {hi }}$ and $\mathrm{H}-2 \mathrm{D}^{\mathrm{b}}$ tetramerhi and then analyzed for annexin $\mathrm{V}$ binding. In vivo blockade of IFN- $\gamma$ and TNF- $\alpha$. RIP-LCMV-NP mice were infected i.p. with $10^{5}$ PFUs of LCMV-Arm on day 0 and with $10^{5}$ PFUs of LCMV-Past 1 month after LCMV-Arm infection. Human TNFR55-IgG1 fusion protein (TNF-blocking reagent; kindly provided by Werner Lesslauer, F. Hoffmann-La Roche Ltd., Basel, Switzerland) was given at $100 \mu \mathrm{g}$ (i.v.) on days 1, 3, 6, and 9 after secondary (Past) infection, and neutralizing rat anti-mouse IFN- $\gamma$ antibody (Pharmingen, San Diego, California, USA) was given at $50 \mu \mathrm{g}$ (i.v.) on days $1,3,6$, and 9 after secondary (Past) infection. Blood glucose was measured in weekly intervals, and values greater than $300 \mathrm{mg} / \mathrm{dl}$ were considered diabetic.

\section{Results}

Prevention of diabetes in NOD and RIP-LCMV mice by a viral infection. To test the hypothesis that virally induced inflammation can disrupt the progression of a prediabetic state to autoimmune disease, we chose the following experimental setup. First, RIP-LCMV-Arm-NP mice were infected i.p. with $10^{5}$ PFUs of LCMV-Arm to initiate T1D. RIP-LCMV-NP mice usually develop diabetes within 2-3 months after the triggering LCMV infection (24). However, when these mice received a secondary infection with LCMV during the prediabetic phase 4 weeks after the autoreactive process had been triggered, diabetes was completely prevented. Interestingly, abrogation of disease did not occur after i.p. injection of $10^{5}$ PFUs of the autologous LCMV strain Armstrong (LCMV-Arm). In contrast, inoculation with the related LCMV strain Pasteur (LCMV-Past) at the same dose ( $10^{5}$ PFUs i.p.) prevented disease in all RIPLCMV-NP mice that were initially infected with LCMVArm (Figure 1a). In both instances, the secondary infections were cleared and abrogation of diabetes was not associated with viral persistence (data not shown). These observations were unexpected, because LCMV strains Arm and Past share a mostly homologous NP sequence and therefore the immunodominant NP epitopes recognized by autoreactive CD4 and CD8 lymphocytes are conserved $(29,34)$. Thus, one would have expected enhancement of disease. Supporting the finding that both LCMV strains can elicit a diabetogenic NP response is the observation that they induced T1D in RIP-LCMV-NP mice, when given as single infections (Figure 1b). The single-infection experiments agree also with earlier reports that showed a correlation between numbers of autoreactive lymphocytes generated after primary LCMV infection of RIP-LCMV-NP mice and severity and incidence of diabetes (35). Here, infection with LCMV-Arm induced a frequency of 1 in 4,000 pCTLs specific for the immunodominant NP epitope $\mathrm{NP}_{396}$, resulting in $95-100 \%$ diabetes, and infection with LCMV-Past induced slightly fewer, 1 in 6,500 $\mathrm{NP}_{396}$-specific pCTLs, resulting in 50\% diabetes (Figure 1b). In contrast, infection with an NP escape variant that was described by us previously (36) induced a frequency that was below the detection limit of 1 in $20,000 \mathrm{NP}_{396}$-specific pCTLs, resulting in no diabetes (Figure 1b). Thus, in the secondary-infection experiments, one would have expected an augmentation of autoimmunity after repriming of LCMV-specific memory lymphocytes by both LCMV strains, but not the abrogation of disease that we found here. Two principal mechanisms came to mind, one involving direct antigenic recognition of autoreactive lymphocytes, the other non-antigen-specific inflammatory processes. We decided to explore both. Thus, we extended our observations to another diabetes model, in order to assess whether abrogation of disease could also occur in the absence of antigenic recognition.

Interestingly, acute LCMV infection was also capable of preventing diabetes in prediabetic NOD mice (Figure 1c). Single i.p. infection of NOD mice at week 10 of age with $10^{5}$ PFUs of LCMV-Arm resulted in a marked reduction of T1D incidence compared with that in uninfected NOD mice (Figure 1c). In addition, late administration of LCMV-Arm, at a time when some of the NOD mice had already developed clinically overt diabetes (week 20 of age), stopped further increase of T1D incidence. However, most of the NOD mice that were diabetic at the time of LCMV-Arm infection remained diabetic to the end of the observation period (Figure 1c), and no reversion of clinical disease occurred.

Administration of $L C M V$ reduces islet infiltration in the RIP$L C M V-N P$ and NOD mouse models. We first examined the target organ histologically to determine the events tak- 
a

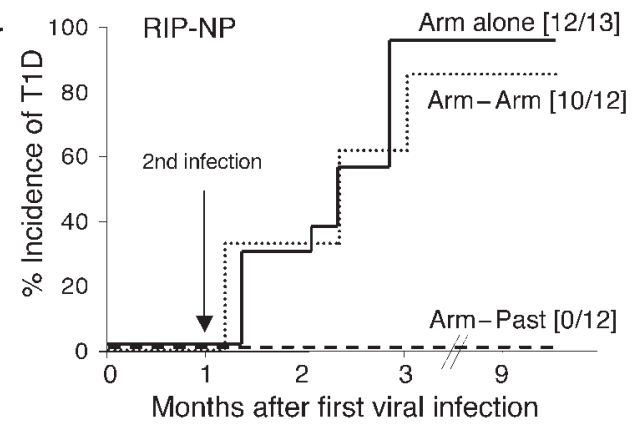

b

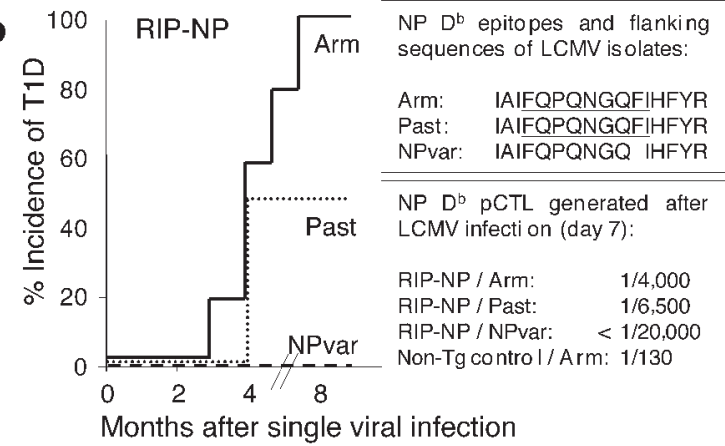

C

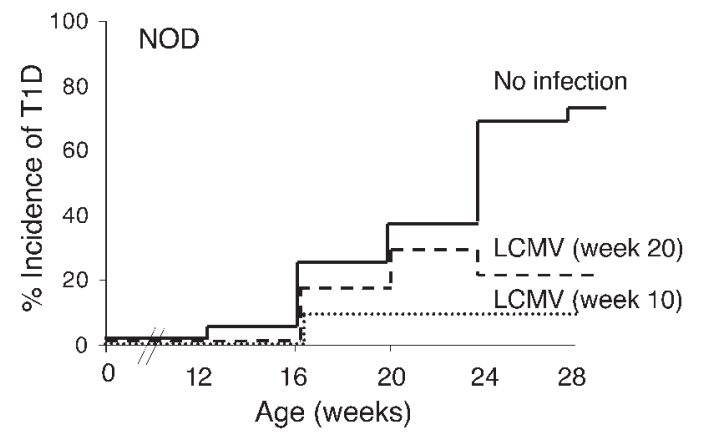

ing place immediately after the abrogative viral infections. As displayed in Figure 2, inoculation of prediabetic RIP-LCMV-NP mice with LCMV-Past was associated with a loss of cellular infiltration that was obvious as early as 3 days after infection (Figure $2 b$, lower panels). In contrast, infection with LCMV-Arm did not have a detectable influence on islet infiltration (Figure $2 \mathrm{~b}$, middle panels), and islets remained heavily infiltrated by both CD8 and CD4 T cells in a pattern similar to that of the infiltration usually found 1 month after primary/initiating LCMV-Arm infection (Figure $2 \mathrm{~b}$, upper panels). The overall degree of islet inflammation was determined in several tissue sections from three or four mice per group using the following scoring system: 0 , no infiltration; 1 , some peri-insular infiltration; 2, heavy peri-insular infiltration with some intra-insular infiltrates; 3 , heavy intra-insular infiltration and/or islet scars (Figure 2c). Mice that received a secondary infection with LCMV-Past showed a reduced overall infiltration of islets (mean score of 0.88 ) when compared with mice that did not receive secondary infection or LCMV-Arm (mean scores of 1.79 and 2.00, respectively). Only very few $(\sim 10 \%)$ of the islets exam-

\section{Figure 1}

Prevention of T1D in NOD and RIP-LCMV mice by LCMV infection given during the prediabetic phase. (a) Cumulative incidence of diabetes in RIP-LCMV-NP mice infected i.p. with $10^{5}$ PFUs of LCMV-Arm on day 0 . One month after infection, mice were infected i.p. with $10^{5}$ PFUs of either LCMV-Arm (Arm-Arm) or LCMV-Past (Arm-Past). Blood glucose was measured in biweekly intervals for at least 9 months, and values greater than $300 \mathrm{mg} / \mathrm{dl}$ were considered diabetic. The number of diabetic mice and the total number of animals used per group are indicated in brackets. (b) Groups of 10-15 RIPLCMV-NP H-2 ${ }^{\mathrm{b}}$ mice were infected i.p. with $10^{5} \mathrm{PFUs}$ of various LCMV strains (single infection). Blood glucose values were determined as described in Methods. The NP escape variant contains a single amino acid change from $F$ to $L$ in position AA404 of the immunodominant $\mathrm{H}-2 \mathrm{D}^{\mathrm{b}} \mathrm{NP}_{396}$ peptide, which prevents binding of the variant $\mathrm{NP}_{396}$ to $\mathrm{H}-2 \mathrm{D}^{\mathrm{b}}$ (36). $\mathrm{pCTL}$ analysis was performed 7 days after infection as described in Methods. Note that RIP-NP mice express the LCMV-NP antigen in their thymus in addition to their islets and therefore have reduced numbers of NP CTLs in their periphery and develop diabetes more slowly, as reported by us previously (24). Non-Tg, nontransgenic C57BL/6 mice. (c) Groups of 10-15 NOD mice were infected i.p. with $10^{5}$ PFUs of LCMV-Arm (single infection) at week 10 or 20 of age. Blood glucose values were determined every 4 weeks.

ined had heavy intra-islet infiltration after LCMV-Past infection (score group 3 ). In contrast, more than $40 \%$ of all islets were heavily infiltrated in LCMV-Arminfected mice and in mice that did not receive a secondary infection (Figure 2c).

A similar exodus of islet-infiltrating lymphocytes was observed in the NOD mouse. Female NOD mice were infected with either LCMV-Arm or LCMV-Past (both i.p. at $10^{5}$ PFUs) at week 9 of age. At day 3 postinfection, pancreata and PDLNs were removed and analyzed for islet infiltration. As displayed in Figure 2, islet infiltration did not progress as much as observed in RIPLCMV mice at week 4 after infection with LCMV (Figure $2 \mathrm{~b}$, upper panels). However, infection with both LCMV-Arm and LCMV-Past reduced islet infiltration by both CD8 and CD4 T cells in the NOD mouse (Figure $2 \mathrm{~b}$, middle and lower panels). The mean insulitis score was reduced from 0.92 in uninfected NOD mice to 0.11 and 0.44 in mice that were infected with LCMVArm or LCMV-Past, respectively (Figure 2c). The rapid egress of lymphocytes from islets in both diabetes models was very striking, and we next examined which events were underlay this phenomenon.

Infection with LCMV induces a chemokine gradient between the PDLN and the pancreas. Chemokines play an important role in the innate defense system against invading microorganisms by attracting lymphocytes and other leukocytes to the site of infection. Among the large family of chemokines, IP-10 (CXCL10) plays a unique role after viral infection of the pancreas. Our previous observations demonstrated that the early and selective expression of IP-10 after infection with LCMV was responsible for the subsequent development of T1D (37). Selective blockade of IP-10 with a neutralizing anti-IP-10 mAb abrogated T1D in the RIP-LCMV mouse (37). Therefore, we used RNase protection assay to analyze the expres- 
a
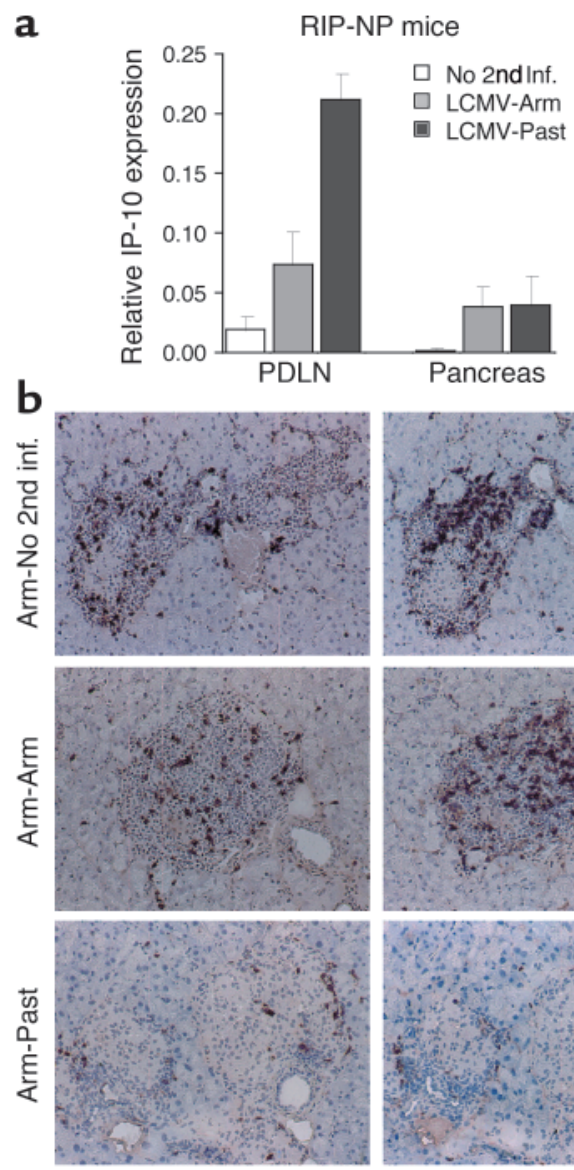

CD8

C

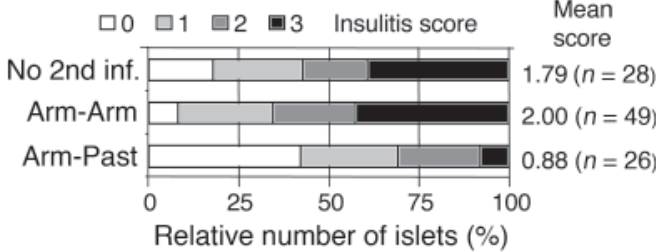

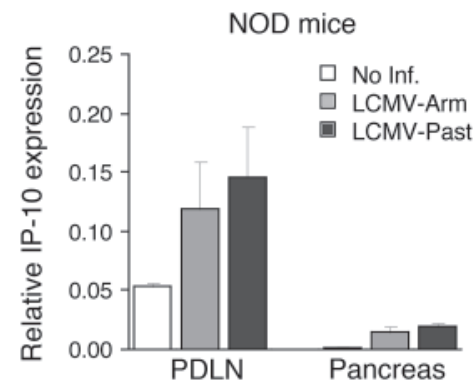
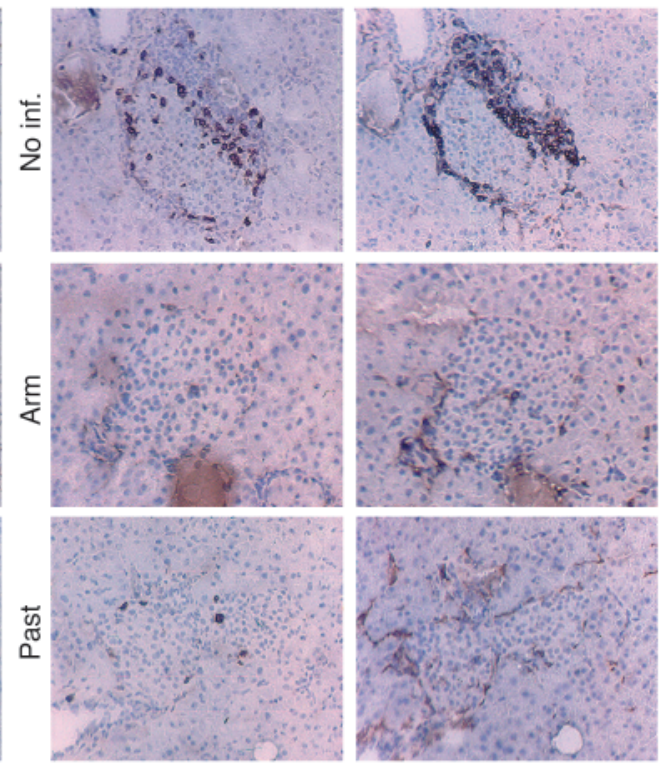

CD8

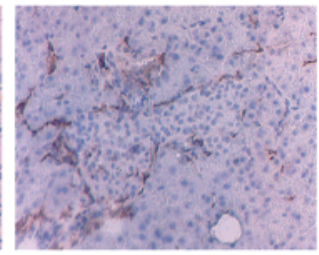

CD4

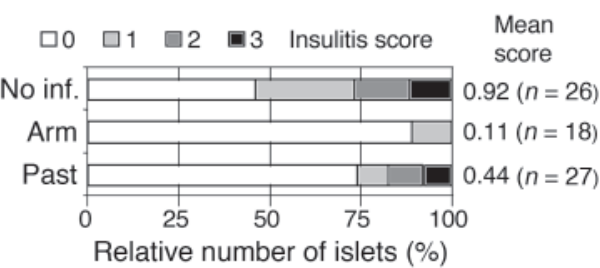

Figure 2

Protective viral infection results in increased IP-10 expression in the PDLN and a decrease of islet-infiltrating CD8 lymphocytes. Left: RIP-LCMVNP mice were infected (inf.) i.p. with $10^{5}$ PFUs of LCMV-Arm and received a secondary i.p. infection (2nd inf.) after 1 month with $10^{5}$ PFUs of LCMV-Arm or LCMV-Past. Right: Nine-week-old NOD mice were infected i.p. with $10^{5}$ PFUs of LCMV-Arm (Arm) or LCMV-Past (Past). (a) Some RIP-LCMV-NP and NOD mice were sacrificed at day 1 after secondary infection for assessment of the expression of IP-10 (CXCR3) in the pancreas and PDLN. The relative IP-10 mRNA expression was analyzed by RNase protection assay and normalized against L32 housekeeping RNA expression $(n=3-4)$. (b) At day 3 after secondary infection, pancreata and PDLNs were harvested, and 6- $\mu \mathrm{m}$ tissue sections were probed for cellular infiltration by CD4 and CD8 T cells. These representative tissue sections show an average degree of islet infiltration per group as found in the pancreas of individual mice $(n=3-4)$. (c) Insulitis score as obtained from sections of 3-4 mice per group. Scoring system: 0, no infiltration; 1, some peri-insular infiltration; 2, heavy peri-insular infiltration with some intra-insular infiltrates; 3 , heavy intra-insular infiltration and/or islet scars. The mean score was obtained by division of the sum of all individual islet-infiltration scores by the total number of islets analyzed.

sion of several chemokines in the pancreas and in the PDLN at day 1 after infection of both NOD and LCMVimmune RIP-NP mice with (secondary) LCMV. Indeed, IP-10 mRNA expression was increased in both pancreas and PDLN of RIP-LCMV-NP mice upon infection with both LCMV-Arm and LCMV-Past. Interestingly, we found a more-than-fivefold higher expression of IP-10 in the PDLN compared with the pancreas (Figure 2a, left panel), indicating the presence of an IP-10 chemokine gradient that increases toward the PDLN. IP-10 expression in the PDLN was highest after infection with LCMV-Past (Figure 2a, left panel), which correlated with reduced islet infiltration and protection from disease. Similar to RIP-LCMV-NP mice, in NOD mice IP-10 mRNA expression was increased upon infection with both LCMV-Arm and LCMV-Past in both pancreas and PDLN, and a much higher expression of IP-10 was detected in the PDLN compared with the pancreas (Fig- 
ure $2 \mathrm{a}$, right panel). An increase of IP-10 expression in the PDLN did not significantly differ between NOD mice that received LCMV-Past or LCMV-Arm (Figure 2a, right panel), which corresponds to the finding that disease is abrogated in both cases.

In contrast to IP-10, none of the other chemokines analyzed - lymphotactin (XCL1), eotaxin (CCL11), MIP-1 $\alpha$ (CCL3), MIP-1 $\beta$ (CCL4), TCA-3 (CCL1), and MCP-1 (CCL2) - was significantly upregulated at day 1 after infection with either LCMV-Past or LCMV-Arm (data not shown). Constitutive expression of RANTES (CCL5) was found in the PDLN, but not in the pancreas, of uninfected NODs and of RIP-LCMV-NP mice that did not receive a secondary infection. However, expression of RANTES was not upregulated after infection with LCMV. Interestingly, our previous findings demonstrated that $\mathrm{CXCR} 3$, the only cellular receptor for IP-10 known to date (38), is indeed expressed on more than $95 \%$ of LCMV-NP 396 -specific CD8 T cells upon LCMV infection (37).

The LCMV-induced IP-10 gradient between the pancreas, where IP-10 was low, and the PDLN, where it was high, suggested that CXCR3-bearing autoaggressive CD8 T cells left the islets and migrated rapidly to the PDLN following abrogative LCMV infection. The higher concentration of IP-10 that abrogative viral infection induced in the PDLN likely caused recruitment of lymphocytes away from the target area (islets), and a reduction of insulitis.

Abrogation of diabetes following LCMV infection is associated with increased apoptosis in the PDLN of NOD and RIP$L C M V$ mice. To further follow the fate of islet-infiltrating cells that appeared to be attracted to the PDLN after LCMV-induced inflammation, we performed TUNEL staining of tissue sections from PDLN. Indeed, we found an increased frequency of apoptotic cells as early as 3 days after the second viral infection with LCMVPast (Figure 3, lower panels). In RIP-LCMV-NP mice that had not received a secondary infection and in those that had received the nonabrogative infection with LCMV-Arm, only a few scattered apoptotic cells could be found. In contrast, abrogative infection with LCMVPast induced typical clusters of apoptotic cells throughout the PDLN (Figure 3, lower left panel). Similar to LCMV-Past-infected RIP-LCMV-NP mice, TUNEL staining of PDLN sections harvested from NOD mice revealed an increased frequency of apoptotic cells as early as 3 days after viral infection with both LCMV-Past and LCMV-Arm (Figure 3, lower right panel). Only a few apoptotic cells were present in PDLN of uninfected NOD mice. In contrast, infection with LCMV-Past induced clusters of apoptotic cells throughout the PDLN in a very similar manner to that observed after secondary infection of RIP-LCMV-NP mice with LCMVPast (Figure 3, lower right panel). Interestingly, even infection with LCMV-Arm induced apoptotic clusters in the NOD mouse, which correlate with the reduced islet infiltration detected in the pancreas of NOD mice infected with LCMV-Arm (Figure 2b). Thus there appears to be a clear correlation between increased apoptosis in the PDLN and prevention of diabetes in both RIP-LCMV and NOD mouse models.

In RIP-LCMV mice, apoptosis of autoaggressive (LCMV-specific) CD8 lymphocytes in the PDLN is enhanced after protective viral infection. One of the advantages of the RIP-LCMV mouse model of T1D is that the target antigen is precisely defined and all the immunodominant and subdominant epitopes for LCMV-NP are known. Therefore, we used the RIP-LCMV model to further dissect the mechanism underlying disruption of the ongoing autoreactive process directly at the level of LCMV-NP 396 -specific (autoaggressive) lymphocytes. The numbers of such autoaggressive CD8 lymphocytes were assessed in the blood, in the spleen, and locally in the PDLN. We compared RIP-LCMV-NP mice that had only received the diabetes-triggering infection $\left(10^{5}\right.$ PFUs i.p.) of either LCMVArm or LCMV-Past with mice that received the initial/primary LCMV-Arm infection followed by an i.p. secondary challenge with $10^{5}$ PFUs of either LCMV-Arm (nonabrogative) or LCMV-Past (abrogative of T1D). As shown in Figure 4a, after undergoing an initial phase of expansion (day 7), LCMV-NP-specific pCTLs were found
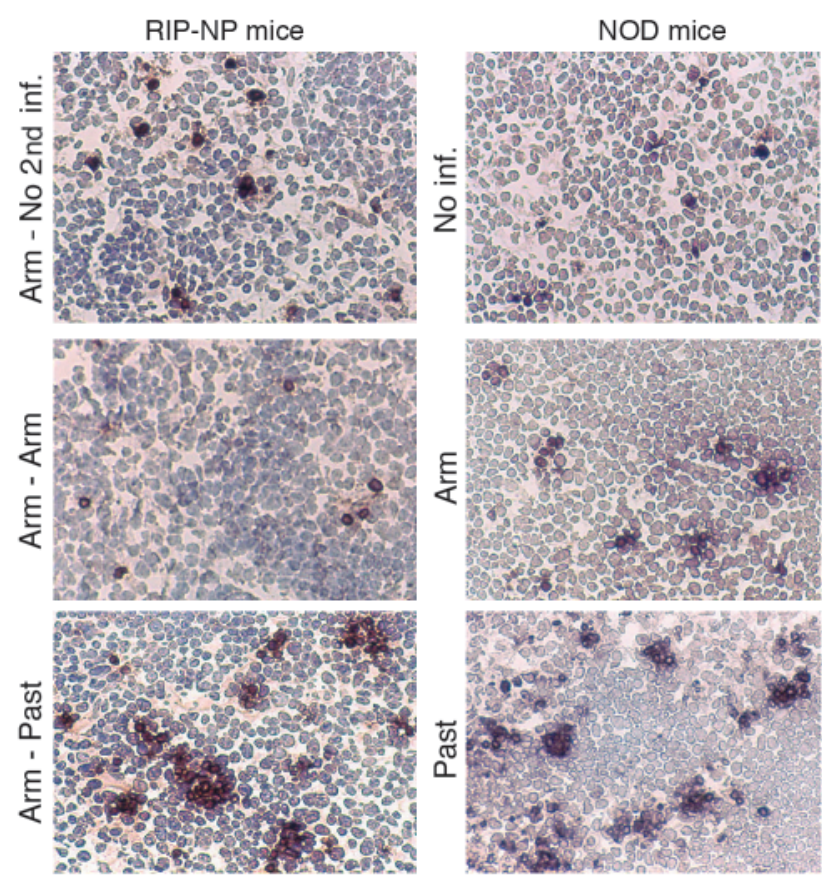

\section{Figure 3}

Abrogation of diabetes following LCMV infection is associated with increased apoptosis in the PDLN of NOD and RIP-LCMV mice. RIP-LCMV-NP mice were infected i.p. with $10^{5}$ PFUs of LCMV-Arm and received a secondary i.p. infection after 1 month with $10^{5}$ PFUs of LCMV-Arm or LCMV-Past. Nine-week-old NOD mice were infected i.p. with $10^{5}$ PFUs of LCMV-Arm or LCMV-Past. At day 3 after infection, PDLNs were harvested, and $6-\mu \mathrm{m}$ tissue sections were probed for apoptotic cells using TUNEL staining. The staining shown represents an average number of apoptotic cells per group as found in individual mice $(n=3-4)$. Note the formation of clusters of apoptotic cells after infection with LCMVPast in both RIP-NP and NOD mice. 

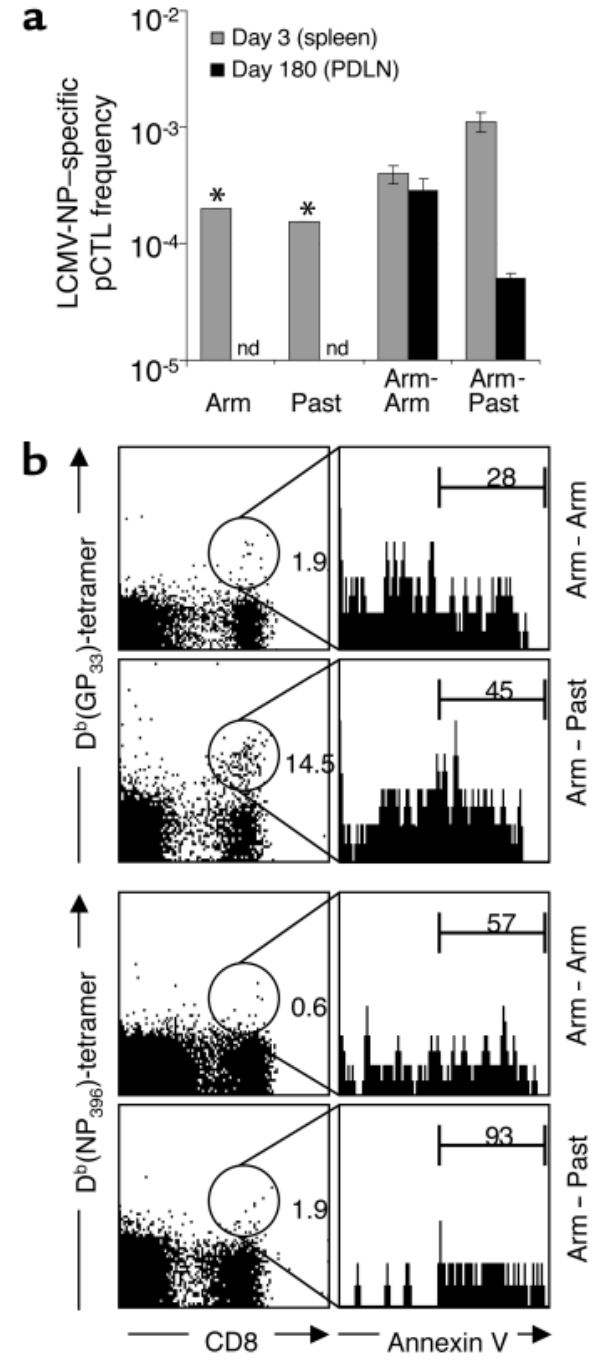

at a significantly reduced level at day 180 postinfection in mice that had received the secondary LCMV-Past infection and were protected from diabetes compared with pCTL frequencies found in nonprotected mice after secondary infection with LCMV-Arm (Figure 4a).

Identification of PDLN CD8 lymphocytes specific for LCMV-NP or LCMV-GP with $\mathrm{D}^{\mathrm{b}}\left(\mathrm{NP}_{396}\right)$ - or $\mathrm{D}^{\mathrm{b}}\left(\mathrm{GP}_{33}\right)$ tetramers, respectively, confirmed the augmented expansion of LCMV-specific CD8 lymphocytes early after secondary infection with LCMV-Past compared with LCMV-Arm (Figure 4b, left panels). However, parallel staining with annexin $V$ revealed a higher frequency of apoptosis in $\mathrm{D}^{\mathrm{b}}\left(\mathrm{NP}_{396}\right)$ - or $\mathrm{D}^{\mathrm{b}}\left(\mathrm{GP}_{33}\right)$-tetramer-positive cells in RIP-LCMV-NP mice that received LCMV-Past (abrogative) compared with those that received LCMV-Arm (nonabrogative) (Figure $4 \mathrm{~b}$, right panels). Thus, increased activation of autoreactive CTLs during an ongoing autoimmune process may lead to their apoptosis and overall disruption of the autoimmune process. At day 180 postinfection, nondiabetic mice that received a secondary infection with LCMVPast had minimal CD8 lymphocytes in their islets and did not develop diabetes during their lifetime. The pro-

\section{Figure 4}

In RIP-LCMV mice, apoptosis of autoaggressive (LCMV-specific) CD8 lymphocytes in the PDLN is enhanced after protective viral infection. (a) Frequency of LCMV-NP-specific PCTLs in RIP-LCMV-NP mice after secondary i.p. infection with $10^{5}$ PFUs of LCMV-Arm or LCMVPast administered 4 weeks after initial LCMV-Arm infection. Note that the reduced $P C T L$ frequency in the PDLN after secondary infection with LCMV-Past correlated with the drastically lower incidence of diabetes in those mice. ${ }^{*} \mathrm{PCTL}$ frequencies in the spleen at day 3 after primary i.p. infection with $10^{5}$ PFUs of LCMV-Arm or LCMV-Past are displayed as a reference. nd, not determined. (b) RIP-LCMV-NP mice were infected i.p. with $10^{5}$ PFUs of LCMV-Arm and, after 1 month, with $10^{5}$ PFUs of either LCMV-Arm or LCMV-Past. At day 3 after secondary infection, PDLN CD8 lymphocytes were stained with anti-CD8 $\mathrm{mAb}, \mathrm{H}-2 \mathrm{D}^{\mathrm{b}}\left(\mathrm{GP}_{33}\right)$ - or $\mathrm{H}-2 \mathrm{D}^{\mathrm{b}}\left(\mathrm{NP}_{396}\right)$-tetramers, and annexin $\mathrm{V}$. CD8 hi, $\mathrm{H}-2 \mathrm{D}^{\mathrm{b}}$-tetramerhi cells (left panels, dot blots) were analyzed with annexin $\mathrm{V}$ for apoptosis (right panels, histograms). The relative numbers of $C D 8^{\text {hi }}, \mathrm{H}-2 \mathrm{D}^{\mathrm{b}}$-tetramerhi cells and annexin $\mathrm{Vhi}^{\text {hi }}$ cells are indicated as percentage of gated cells.

tection from diabetes was not caused by regulatory lymphocytes, since it was not transferable (data not shown) and no significant differences in Th1/Th2 regulatory cytokines, such as IFN- $\gamma$, IL-4, and IL-10, were found in pancreata of doubly infected mice without diabetes (RNase protection analysis; data not shown).

High load of viral antigen correlates with disruption of the autoimmune process in RIP-LCMV mice. Since, in RIP-LCMV mice as opposed to NOD mice, a direct antigenic crossreactivity was given between the abrogative viral strain and the $\beta$ cell autoantigen (LCMV-NP), we asked whether a direct antigen-specific component might play an important role in the diabetes prevention that we had observed after LCMV-Past, but not LCMV-Arm, infection. Furthermore, both strains prevented disease in the NOD mice, whereas only LCMV-Past prevented disease in RIP-LCMV mice. One possibility for this discrepancy between NOD and RIP-LCMV mice was a difference in viral load and the associated difference in viral antigen as well as inflammation in the individual organs involved. Indeed, $\mathrm{T}$ lymphocyte relocalization and disease abrogation after LCMV infection of prediabetic mice correlated with higher viral titers detected in the pancreas, the spleen, and the PDLN 3 days after inoculation. Challenge with LCMV-Past resulted in viral titers ranging from $3 \times 10^{5} \mathrm{PFUs} / 10 \mathrm{mg}$ tissue (pancreas) up to $3 \times 10^{6}$ PFUs/10 mg tissue (PDLN) (Figure 5). In contrast, i.p. infection with $10^{5}$ PFUs of LCMV-Arm that did not prevent diabetes yielded viral titers that were up to three orders of magnitude lower (Figure 5). The marked differences in the viral load between primary and secondary LCMV-Arm infection are most likely a direct consequence of LCMV-Arm immunity occurring after primary infection of RIP-LCMV mice, resulting in accelerated LCMV-Arm clearance. In accordance with this finding, LCMV-NP ${ }_{396}$-specific autoreactive CTLs were activated and expanded more strongly after secondary infection with LCMV-Past (Figure 4a) but exhibited TNF- $\alpha$ and IFN- $\gamma$ production similar to that observed after secondary LCMV-Arm infection. 


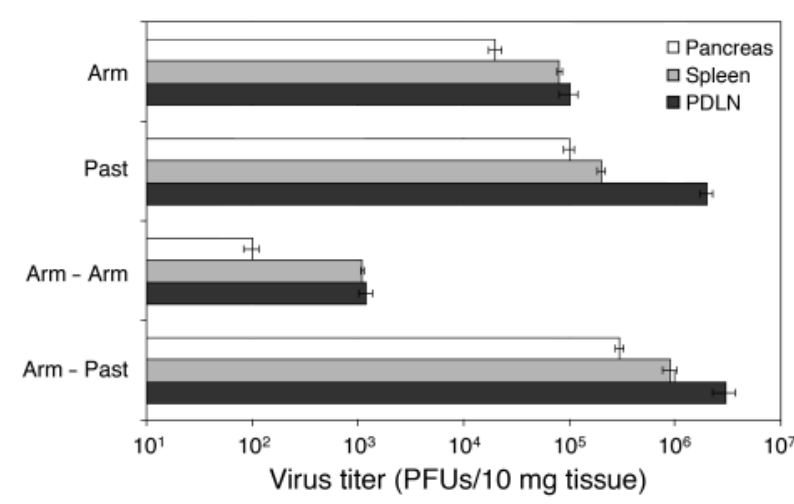

Figure 5

Abrogative infection of RIP-LCMV mice with LCMV-Past results in decreased $P C T L$ frequencies and higher viral titers in the PDLN. Viral titers were determined in the pancreas, the spleen, and the PDLN of RIP-LCMV-NP mice at day 3 after primary i.p. infection with $10^{5}$ PFUs of LCMV-Arm or LCMV-Past or, alternatively, at day 3 after secondary infection (administered 4 weeks after initial LCMV-Arm infection) with $10^{5}$ PFUs of LCMV-Arm (nonabrogative) or LCMV-Past (abrogates diabetes). Data are shown as mean PFUs/10 mg tissue $( \pm \mathrm{SD})$ as obtained from plaque assays $(n=4)$.

Blockade of IFN- $\gamma$ or TNF- $\alpha$ prevents abrogation of diabetes by secondary viral infection in RIP-LCMV mice. To examine whether cytokines, such as TNF- $\alpha$ or IFN- $\gamma$, that are known to play an important role in initiating and/or maintaining an inflammatory process were required for the abrogation of diabetes after a second viral infection, we immunized RIP-LCMV-NP mice with $10^{5}$ PFUs LCMV-Past (i.p.) 1 month after initiation of diabetes with LCMV-Arm and then blocked TNF- $\alpha$ or IFN- $\gamma$ systemically with specific blocking agents. Both the TNF receptor IgG1 fusion protein and the antibody to IFN- $\gamma$ had been shown to neutralize these cytokines on a systemic level $(39,40)$ and had clear in vivo activity as evidenced by their ability to prevent LCMVinduced diabetes in our RIP-LCMV-GP model system for rapid-onset T1D $(24,41)$. In the present study, RIPLCMV-NP mice were treated with the blocking reagents on days 1, 3, 6, and 9 after the abrogative LCMV-Past infection. As shown in Figure 6, blockade of either TNF- $\alpha$ or IFN- $\gamma$ prevented the abrogative effect of the second viral infection, and diabetes developed in the majority of mice (Figure 6a). Thus, cytokines with predominantly proinflammatory functions (36) can also have beneficial effects during an ongoing autoimmune process. In this scenario they appear to be involved in enhancing apoptosis of autoaggressive lymphocytes after their initial activation.

In vitro blockade of TNF- $\alpha$ but not IFN- $\gamma$ prevents apoptosis of activated, antoaggressive CD8 T cells. We further sought to investigate whether TNF- $\alpha$ and/or IFN- $\gamma$ could play a direct role in the destruction of autoaggressive CD8 T cells in vitro. We harvested the spleen and the PDLN of C57BL/ 6 mice at days 7 and 30 after LCMV-Arm infection, stimulated the cells overnight with the immun- odominant $\mathrm{NP}_{396}$ peptide in the presence or absence of blocking reagents for TNF- $\alpha$ (TNFR55-IgG1) or IFN- $\gamma$ (anti-IFN- $\gamma \mathrm{mAb}$ ), and analyzed the frequency of apoptotic $\mathrm{D}^{\mathrm{b}}\left(\mathrm{NP}_{396}\right)$-specific $\mathrm{CD} 8 \mathrm{~T}$ cells using annexin $\mathrm{V}$ staining. Blocking of TNF- $\alpha$ resulted in a drastically reduced frequency of apoptotic $\mathrm{D}^{\mathrm{b}}\left(\mathrm{NP}_{396}\right)$-specific $\mathrm{CD} 8$ $\mathrm{T}$ cells isolated from both the spleen and the PDLN (Figure 6b). In contrast, no reduction was observed when IFN- $\gamma$ was neutralized, indicating that, in vivo, IFN- $\gamma$ may act through a more indirect pathway whereas TNF- $\alpha$ may increase apoptosis directly. Interestingly, in CD8 cells, reduction in apoptosis was only observed in the PDLN, not in the spleen, even though the frequency of $\mathrm{D}^{\mathrm{b}}\left(\mathrm{NP}_{396}\right)$-specific $\mathrm{CD} 8 \mathrm{~T}$ cells was similar in both organs (Figure 6b). This suggests that the local inflammation was more dependent on TNF- $\alpha$ in the PDLN than in the spleen.

\section{Discussion}

Viruses that do not directly destroy $\beta$ cells can enhance the course of autoimmune diabetes through several different and distinct pathways. The first acts directly on autoreactive lymphocytes through either cross-reactivity $(19,20)$ or cytokine-mediated bystander effects $(18)$, which respectively can either increase their numbers or reduce them by induction of apoptosis (42). The second affects $\beta$ cell death via the local milieu of cytokines in the pancreas. However, our study shows that an experimental cure from disease following viral infection is possible by recruitment of autoaggressive $T$ cells away from the islet infiltrate. This involves virally induced inflammation in the PDLN, most notably a strong induction of IP-10 that is known to predominantly attract activated Th1type T lymphocytes expressing CXCR3 $(38,43)$. The attraction of infiltrating lymphocytes to the pancreatic node is associated with increased $\mathrm{T}$ cell apoptosis. This disruption of the autoaggressive process is irreversible in the sense that the $T$ cells, once they leave the islets, will not return, resulting in long-lasting prevention of disease in both NOD and RIP-LCMV mice.

In the NOD model, the observed recruitment away from islets is most likely antigen independent, since LCMV is not known to cross-react with any of the major $\beta$ cell autoantigens in NOD mice, such as insulin, glutamic acid decarboxylase, and islet antigen 2 (IA2). However, the LCMV-induced abrogation of disease has a permanent effect, despite the fact that the autoaggressive response in the NOD model is thought to be present throughout life. Interestingly, it was recently reported that expression of IP-10 is found in islets of NOD mice and that its magnitude increases with age (44). Thus, expression of IP-10 may usually act as an islet-retention factor for autoaggressive $T$ lymphocytes. Infection with LCMV and the associated high level of IP-10 expression outside the islets (in the PDLN) would thus permanently disrupt this retention and recruit $\mathrm{T}$ lymphocytes away from the islets. We previously showed that, after LCMV infection, more than $80 \%$ of all CD8 T lymphocytes express high levels of CXCR3 (IP-10 receptor) on their cell 

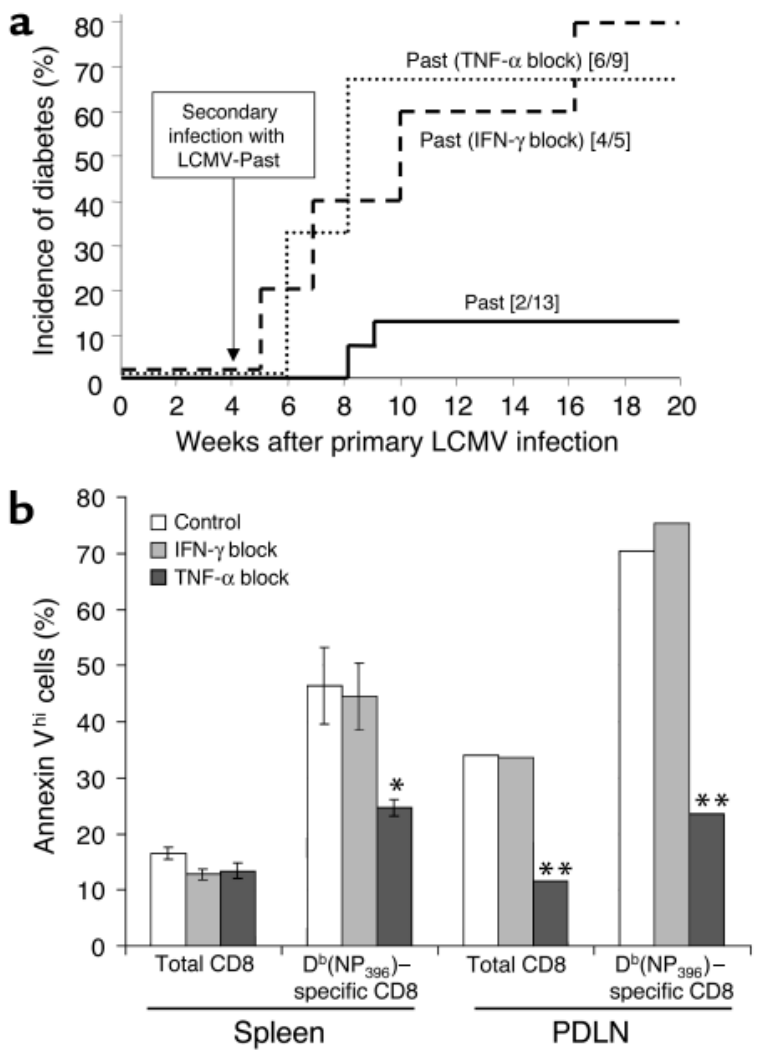

surface (37). One could speculate that the reduction of cellular infiltration in the islets after abrogative viral infection will sufficiently change the inflammatory milieu locally in the islets to prevent future infiltration by newly activated autoreactive lymphocytes.

In contrast to the NOD model, abrogation of T1D by LCMV infection in RIP-LCMV-NP mice has an additional, antigen-specific component. Just as in NOD mice, the recruitment of autoaggressive $T$ lymphocytes away from the islets is associated with an IP-10 gradient between the pancreas and the PDLN. However, viral growth seems to influence the abrogative process in at least two ways. First, depending on the viral titer and the associated local inflammation in the PDLN, the IP-10 gradient was steeper, resulting in a more effective lymphocyte relocalization process. This finding does not stand in contrast with our recent report demonstrating a central role of IP-10 in the attraction of LCMV-specific T lymphocytes and its importance for the induction of autoimmune disease (37), if one considers several crucial aspects of the timing and precise localization of IP10 expression after LCMV infection. After primary infection of RIP-LCMV-NP mice with LCMV, IP-10 is first elevated in the PDLN (day 1 postinfection) and only later on in the pancreas (days 4-10), suggesting that $\mathrm{T}$ cells are first attracted to the PDLN and only later migrate to the pancreas (U. Christen, unpublished observations). However, after secondary (abrogative) infection with LCMV, such T cells, which are already in the islets and activated, are first attracted to the PDLN in an IP-10-dependent manner, as shown in the present

\section{Figure 6}

Blockade of IFN- $\gamma$ or TNF- $\alpha$ prevents abrogation of diabetes by secondary viral infection. (a) Cumulative incidence of diabetes in RIPLCMV-NP mice infected i.p. with $10^{5}$ PFUs of LCMV-Arm on day 0 and $10^{5}$ PFUs of LCMV-Past 1 month after LCMV-Arm infection. Blood glucose was measured in weekly intervals, and values greater than $300 \mathrm{mg} / \mathrm{dl}$ were considered diabetic. The number of diabetic mice and the total number of animals used per group are indicated in brackets. Human TNFR55-IgG1 fusion protein $(38,39)$ or neutralizing rat anti-mouse IFN- $\gamma$ antibody (Pharmingen, San Diego, California, USA) were given at $100 \mu \mathrm{g}$ (i.v.) or $50 \mu \mathrm{g}$ (i.v.), respectively, at days $1,3,6$, and 9 after secondary (Past) infection. (b) In vitro hyperstimulation with $\mathrm{D}^{\mathrm{b}}\left(\mathrm{NP}_{396}\right)$-tetramers $\left(16\right.$ hours at $\left.37^{\circ} \mathrm{C}\right)$ of lymphocytes isolated from spleen and PDLN of C57BL/ 6 mice at 1 week after LCMV-Arm infection. Apoptosis of $\mathrm{D}^{\mathrm{b}}\left(\mathrm{NP}_{396}\right)$-specific CD8 $T$ cells was determined by staining with annexin $V$-phycoerythrin conjugate in parallel to staining with $\mathrm{D}^{\mathrm{b}}\left(\mathrm{NP}_{396}\right)$-tetramers. Cells were incubated in medium alone (control) or in the presence of human TNFR55-IgG1 fusion protein $(100 \mu \mathrm{g} /$ well) or neutralizing rat anti-mouse IFN- $\gamma$ antibody $(100 \mu \mathrm{g} /$ well $)$. ${ }^{*}$ Among splenocytes, significant differences in the number of $\mathrm{D}^{\mathrm{b}}\left(\mathrm{NP}_{396}\right)$-specific CD8 T cells

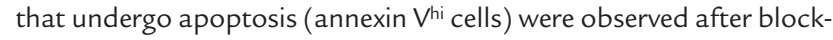
ade of TNF- $\alpha$ compared with the control stimulation, but not after IFN- $\gamma$ blockade (Student's $t$ test, $P<0.05$ ). ${ }^{*}$ PDLNs were collected from three mice, and cells were pooled in order to increase the number of $\mathrm{D}^{\mathrm{b}}\left(\mathrm{NP}_{396}\right)$-specific CD8 T cells within the experimental setup.

study. Afterward, they are unable to re-enter into the pancreas, because the majority of them have been eliminated in the PDLN by apoptosis.

Second, in addition to local inflammation, the viral titer influences the antigenic load in the PDLN. The correlation of viral titer (antigen load) in the PDLN with abrogation of disease suggests an antigen-driven induction of apoptosis of autoaggressive $\mathrm{T}$ lymphocytes. Because of the lack of the respective antigens, this second mechanism is not likely to be operative in the NOD model. In the RIP-LCMV system, the high (self-viral) antigenic load after secondary infection (Figure 5) is a prerequisite for the abrogative effect, since lower local viral titers of LCMV, as observed after secondary infection with LCMV-Arm, do not affect the disease course, although antigenic recognition of immunodominant CD4 and CD8 NP epitopes is shared between LCMVArm and LCMV-Past strains (Figure 1). Thus, activation of autoreactive lymphocytes can have either detrimental or beneficial effects, and the fate of the ongoing autoimmune process is decided by the precise kinetics of the autoimmune response and the presence of inflammatory cytokines, such as TNF- $\alpha$ or IFN- $\gamma$, elicited by a viral infection. Although we found that the clearance of LCMV-Arm was accelerated in LCMV-Arm mice compared with naive mice (Figure 5), the viral titer of LCMV-Past was similar in LCMV-Arm-immune and naive mice (Figure 5). Thus, heterologous immunity, suggested to occur after sequential infections by heterologous viruses, $(45,46)$ seems to play only a minor role after sequential infection with LCMV-Arm followed 
by LCMV-Past. More important here is that viral infections with variants of the same strain might exhibit differential effects, because their ability to grow to different viral titers will influence their effect on ongoing disease. Since many RNA viruses occur as quasispecies harboring a multitude of different sequences, mutating relatively rapidly and selected for fitness in vivo (47), the likelihood of a specific viral strain causing T1D or MS in individuals at risk will be difficult to predict or record with immunological and virological analyses.

Mechanistically, our in vivo observations with blockade of TNF- $\alpha$ or IFN- $\gamma$ demonstrate the importance of both cytokines in the abrogation of the prediabetic process, since they are required for induction of apoptosis of autoaggressive CD8 T lymphocytes in RIP-LCMV mice. However, TNF- $\alpha$ appears to mediate this in a more direct fashion, since the proapoptotic effect can be demonstrated directly in vitro using cultures of activated, autoaggressive LCMV-NP lymphocytes. In contrast, based on our findings presented here, we propose that IFN- $\gamma$ is more indirectly connected to apoptosis of autoaggressive T cells, because addition of IFN- $\gamma$ to in vitro cultures has no detectable effect. Most likely, IFN- $\gamma$ acts during the brief activation and expansion of the autoaggressive response following the abrogative viral infection, for example by upregulating MHC class I. Indeed, IFN- $\gamma$ is usually the earliest cytokine produced, for example by NK cells, after LCMV infection, whereas TNF- $\alpha$ peaks later and is mostly generated by CD 8 lymphocytes and certain APCs.

One can conclude that certain proinflammatory cytokines and chemokines can have beneficial effects during the later course of an ongoing autoimmune process. Similarly, Furlan et al. demonstrated a protective effect of IFN- $\gamma$ when delivered intrathecally using a herpes simplex virus-1-derived vector containing the mouse IFN- $\gamma$ gene, in a mouse model for the chronicprogressive form of experimental autoimmune encephalomyelitis (EAE) (48). Recovery of IFN- $\gamma$-treated mice after initiation of EAE by immunization with myelin oligodendrocyte glycoprotein ${ }_{35-55}\left(\mathrm{MOG}_{35-55}\right)$ was associated with an increased rate of apoptosis of CNS-infiltrating lymphocytes (48). Thus, the precise location, dose, and timing of cytokine expression will be essential for achieving a reduction of the autoaggressive response. Indeed, a similar scenario can occur for TNF- $\alpha$. The exact time of $\beta$ cell-restricted TNF- $\alpha$ expression determines whether or not autoimmune diabetes develops in RIP-LCMV-GP mice $(24,41)$. Alternatively, under the appropriate circumstances early in disease, autoimmune diabetes is dependent on IFN- $\gamma(36)$, as well as TNF- $\alpha$, and blockade of either molecule prevents disease $(24,36,41)$.

Our findings could offer an explanation of why it has been very difficult to establish a causal relationship between viruses and T1D, and maybe why autoimmune diseases such as diabetes occur with lower incidence in countries with poorer hygienic/microbial control. It has been noted that development of diabetes is reduced in the presence of higher amounts of pathogens, as is the case in equatorial countries and, for example, in some less hygienic vivariums housing NOD mice. Certainly, our data show that an ongoing or programmed autoimmune process can be aborted or diminished if induction of chemokines such as IP-10, as well as inflammatory cytokines, in the vicinity of the pancreatic islets recruits lymphocytes away from the autoaggressive process, leading to their demise. The precise timing and localization of the cytokines and chemokines is crucial and must be factored in when one is considering the complex links between inflammation and autoimmunity.

\section{Acknowledgments}

This is publication no. 453 from the Division of Immune Regulation, La Jolla Institute for Allergy and Immunology. M.G. von Herrath was supported by NIH grants R01AI4415, R29DK51091, and AI41439 and by the Juvenile Diabetes Research Foundation, International Career Development Award no. 296120. M.B.A. Oldstone was supported by NIH grant AI41439, and U. Christen was supported by a fellowship from the Juvenile Diabetes Research Foundation (3-2000-510) and by a fellowship from the Swiss Foundation for MedicalBiological Grants.

1. Redondo, M.J., et al. 1999. Genetic determination of islet cell autoim munity in monozygotic twin, dizygotic twin, and non-twin siblings of patients with type 1 diabetes: prospective twin study. BMJ. 318:698-702.

2. Kallmann, B.A., et al. 1999. Cytokine secretion patterns in twins discordant for Type I diabetes. Diabetologia. 42:1080-1085.

3. Thomson, G., et al. 1988. Genetic heterogeneity, modes of inheritance, and risk estimates for a joint study of Caucasians with insulin-dependent diabetes mellitus. Am. J. Hum. Genet. 43:799-816.

4. Honeyman, M.C., et al. 2000. Association between rotavirus infection and pancreatic islet autoimmunity in children at risk of developing type 1 diabetes. Diabetes. 49:1319-1324.

5. Notkins, A.L., and Yoon, J.-W. 1984. Virus-induced diabetes mellitus. In Concepts in viral pathogenesis. A.L. Notkins and M.B.A. Oldstone, editors. Springer-Verlag. New York, New York, USA. 241-247.

6. Yoon, J.W., Ihm, S.H., and Kim, K.W. 1989. Viruses as a triggering factor of type 1 diabetes and genetic markers related to the susceptibility to the virus-associated diabetes. Diabetes Res. Clin. Pract. 7(Suppl. 1):S47-S58.

7. Notkins, A.L., Yoon, J.-W., Onodera, T., Toniolo, A., and Jenson, A.B. 1981. Virus-induced diabetes mellitus. Perspectives in Virology. 11:141-162.

8. von Herrath, M.G., Holz, A., Homann, D., and Oldstone, M.B. 1998. Role of viruses in type I diabetes. Semin. Immunol. 10:87-100.

9. von Herrath, M.G. 2000. Obstacles to identifying viruses that cause autoimmune disease. J. Neuroimmunol. 107:154-160.

10. Oldstone, M.B. 1990. Viruses as therapeutic agents. I. Treatment of nonobese insulin-dependent diabetes mice with virus prevents insulindependent diabetes mellitus while maintaining general immune competence. J. Exp. Med. 171:2077-2089.

11. Oldstone, M.B. 1988. Prevention of type I diabetes in nonobese diabetic mice by virus infection. Science. 239:500-502.

12. Oldstone, M.B., Southern, P., Rodriquez, M., and Lampert, P. 1984. Virus persists in beta cells of islets of Langerhans and is associated with chemical manifestations of diabetes. Science. 224:1440-1443.

13. Rodriguez, M., Garrett, R.S., Raitt, M., Lampert, P.W., and Oldstone, M.B. 1985. Virus persists in beta cells of islets of Langerhans and infection is associated with chemical manifestations of diabetes. II. Morphologic observations. Am. J. Pathol. 121:497-504.

14. Guberski, D.L., et al. 1991. Induction of type I diabetes by Kilham's rat virus in diabetes-resistant BB/Wor rats. Science. 254:1010-1013.

15. Tishon, A., and Oldstone, M.B. 1987. Persistent virus infection associated with chemical manifestations of diabetes. II. Role of viral strain, environmental insult, and host genetics. Am. J. Pathol. 126:61-72.

16. Schwimmbeck, P.L., Dyrberg, T., and Oldstone, M.B. 1988. Abrogation of diabetes in BB rats by acute virus infection. Association of viral-lymphocyte interactions. J. Immunol. 140:3394-3400.

17. Klavinskis, L.S., Notkins, A.L., and Oldstone, M.B. 1988. Persistent viral infection of the thyroid gland: alteration of thyroid function in the 
absence of tissue injury. Endocrinology. 122:567-575.

18. Horwitz, M.S., et al. 1998. Diabetes induced by Coxsackie virus: initiation by bystander damage and not molecular mimicry. Nat. Med. 4:781-785.

19. Wucherpfennig, K.W., and Strominger, J.L. 1995. Molecular mimicry in $\mathrm{T}$ cell-mediated autoimmunity: viral peptides activate human $\mathrm{T}$ cell clones specific for myelin basic protein. Cell. 80:695-705.

20. Atkinson, M.A., et al. 1994. Cellular immunity to a determinant common to glutamate decarboxylase and coxsackie virus in insulin-dependent diabetes. J. Clin. Invest. 94:2125-2129.

21. Makino, S., et al. 1980. Breeding of a non-obese, diabetic strain of mice. Jikken Dobutsu. 29:1-13.

22. Ohashi, P., et al. 1991. Ablation of tolerance and induction of diabetes by virus infection in viral antigen transgenic mice. Cell. 65:305-317.

23. Oldstone, M.B.A., Nerenberg, M., Southern, P., Price, J., and Lewicki, H. 1991. Virus infection triggers insulin-dependent diabetes mellitus in a transgenic model: role of anti-self (virus) immune response. Cell. 65:319-331.

24. von Herrath, M.G., Dockter, J., and Oldstone, M.B.A. 1994. How virus induces a rapid or slow onset insulin-dependent diabetes mellitus in a transgenic model. Immunity. 1:231-242.

25. Homann, D., et al. 1999. Autoreactive CD4+ T cells protect from autoimmune diabetes via bystander suppression using the IL-4/Stat 6 pathway. Immunity. 11:463-472.

26. Gairin, J.E., Mazarguil, H., Hudrisier, D., and Oldstone, M.B. 1995. Optimal lymphocytic choriomeningitis virus sequences restricted by $\mathrm{H}-2 \mathrm{Db}$ major histocompatibility complex class I molecules and presented to cytotoxic T lymphocytes. J. Virol. 69:2297-2305.

27. Oxenius, A., Zinkernagel, R.M., and Hengartner, H. 1998. Comparison of activation versus induction of unresponsiveness of virus-specific CD4+ and CD8+ T cells upon acute versus persistent viral infection. Immunity. 9:449-457.

28. van der Most, R.G., et al. 1998. Identification of Db- and Kb-restricted subdominant cytotoxic T-cell responses in lymphocytic choriomeningitis virus-infected mice. Virology. 240:158-167.

29. Dutko, F.J., and Oldstone, M.B. 1983. Genomic and biological variation among commonly used lymphocytic choriomeningitis virus strains. J. Gen. Virol. 64:1689-1698.

30. Lewicki, H., et al. 1995. CTL escape viral variants. I. Generation and molecular characterization. Virology. 210:29-40.

31. von Herrath, M.G., Guerder, S., Lewicki, H., Flavell, R., and Oldstone, M.B.A. 1995. Coexpression of B7.1 and viral (self) transgenes in pancreatic $\beta$-cells can break peripheral ignorance and lead to spontaneous autoimmune diabetes. Immunity. 3:727-738

32. Garboczi, D.N., et al. 1996. Assembly, specific binding, and crystallization of a human TCR-alphabeta with an antigenic Tax peptide from human T lymphotropic virus type 1 and the class I MHC molecule HLAA2. J. Immunol. 157:5403-5410.

33. Busch, D.H., Pilip, I.M., Vijh, S., and Pamer, E.G. 1998. Coordinate reg- ulation of complex $T$ cell populations responding to bacterial infection. Immunity. 8:353-362.

34. Southern, P.J., et al. 1987. Molecular characterization of the genomic S RNA segment from lymphocytic choriomeningits virus. Virology. 157:145-155.

35. Sevilla, N., et al. 2000. Virus-induced diabetes in a transgenic model: role of cross-reacting viruses and quantitation of effector T cells needed to cause disease. J. Virol. 74:3284-3292.

36. von Herrath, M.G., and Oldstone, M.B.A. 1997. Interferon- $\gamma$ is essential for destruction of $\beta$-cells and development of insulin-dependent diabetes mellitus. J. Exp. Med. 185:531-539.

37. Christen, U., McGavern, D., Luster, A.D., von Herrath, M.G., and Oldstone, M.B.A. 2003. Among CXCR3 chemokines, IP-10 (CXCL10) but not Mig (CXCL9) imprints a pattern for the subsequent development of autoimmune disease. J. Immunol. 171:6838-6845.

38. Loetscher, M., et al. 1996. Chemokine receptor specific for IP10 and Mig: structure, function, and expression in activated lymphocytes. J. Exp. Med. 184:963-969.

39. Mori, L., Iselin, S., De Libero, G., and Lesslauer, W. 1996. Attenuation of collagen-induced arthritis in 55-kDa TNF receptor type 1 (TNFR1)IgG1-treated and TNFR1-deficient mice. J. Immunol. 157:3178-3182.

40. Lesslauer, W. 1998. Fc fusion proteins: TNF $\alpha$ receptor IgG, design principles and activities. In Antibody fusion proteins. S. Chamow and A. Ashkenazi, editors. John Wiley \& Sons. New York, New York, USA. 251-281.

41. Christen, U., et al. 2001. A dual role for TNF-alpha in type 1 diabetes: islet-specific expression abrogates the ongoing autoimmune process when induced late but not early during pathogenesis. J. Immunol. 166:7023-7032

42. Abbas, A.K. 1996. Die and let live: eliminating dangerous lymphocytes Cell. 84:655-657.

43. Qin, S., et al. 1998. The chemokine receptors CXCR3 and CCR5 mark subsets of $\mathrm{T}$ cells associated with certain inflammatory reactions. J. Clin. Invest. 101:746-754

44. Cardozo, A.K., et al. 2003. IL-1beta and IFN-gamma induce the expression of diverse chemokines and IL-15 in human and rat pancreatic islet cells, and in islets from pre-diabetic NOD mice. Diabetologia. 46:255-266.

45. Brehm, M.A., et al. 2002. T cell immunodominance and maintenance of memory regulated by unexpectedly cross-reactive pathogens. Nat. Immunol. 3:627-634.

46. Selin, L.K., et al. 1999. Attrition of T cell memory: selective loss of LCMV epitope-specific memory CD8 T cells following infections with heterologous viruses. Immunity. 11:733-742.

47. Domingo, E., et al. 1985. The quasispecies (extremely heterogeneous) nature of viral RNA genome populations: biological relevance. A review. Gene. 40:1-8.

48. Furlan, R., et al. 2001. Intrathecal delivery of IFN-gamma protects C57BL/6 mice from chronic- progressive experimental autoimmune encephalomyelitis by increasing apoptosis of central nervous systeminfiltrating lymphocytes. J. Immunol. 167:1821-1829. 